\title{
Supporting Information for "The Handedness of DNA Assembly Around Carbon Nanotubes Is Determined by the Chirality of DNA"
}

\author{
Gül H. Zerze, ${ }^{\dagger}$ Frank H. Stillinger,,+ and Pablo G. Debenedetti*, ${ }^{* \dagger}$ \\ $\dagger$ Department of Chemical and Biological Engineering, Princeton University, Princeton, \\ New Jersey 08544, USA \\ $\ddagger$ Department of Chemistry, Princeton University, Princeton, New Jersey 08544, USA \\ E-mail: pdebene@princeton.edu
}

\section{Simulation Methods}

\section{Modeling of CNT}

Initial coordinates of all carbon nanotube structures are generated using Nanotube Builder of Visual Molecular Dynamics (VMD) program ${ }^{1}$ where C-C distance is taken as $0.1418 \mathrm{~nm}$ and CNTs are extended to the edges (of a cubic box). CNT atoms are modeled as a standard sp2-hybridized carbon for non-bonded interactions. CNT atoms are kept rigid during the course of simulations.

\section{Modeling of DNA}

Chirality of the DNA structures are imposed as the initial condition. Initial coordinates of all right-handed, single-stranded DNA structures are generated using the internal coordinates 
table of the CHARMM36 force field for the CHARMM program. ${ }^{2}$ For the left handed DNA structures, we build an internal coordinates table for each RNA/DNA residue and used these tables to generate the initial coordinates of left-handed, single-stranded DNA structures, using the CHARMM program. CHARMM36 force field (with TIP3P water) is used to model the DNA strands. The force field for D-nucleotides is transferable to L-nucleotides for all the bonded and non-bonded parameters. The asymmetric torsional terms would be affected from inverted chirality but we note that there is not any asymmetric dihedrals in this force field.

For each system, an extended DNA configuration is placed near given CNT chirality and system is solvated to achieve $\sim 1000 \mathrm{~kg} / \mathrm{m}^{3}$ water density outside CNT. 17 counter-ions (Na+) is placed to provide electroneutrality of the system. Coordinates are energy-minimized and equilibrated in an NVT $(\mathrm{T}=300 \mathrm{~K})$ simulation. Production simulations are performed in the NVT ensemble. An example initial condition is illustrated in Figure S3.

\section{Advanced-Sampling}

We perform parallel tempering in the well-tempered ensemble (PTWTE) simulations ${ }^{3-5}$ of the CNT-DNA systems for at least $550 \mathrm{~ns} /$ replica using 14 replicas. In the framework of PTWTE, the potential energy is biased using $500 \mathrm{~kJ} / \mathrm{mol}$ Gaussian width, $1.0 \mathrm{~kJ} / \mathrm{mol}$ initial Gaussian height, with Gaussian potentials added every 2000 steps, with a bias factor of 20 . Prior to starting the PTWTE simulations, unbiased simulations at each replica temperature are performed for $200 \mathrm{ps}$ in order to equilibrate the potential energy of the replicas. Temperatures of the 14 replicas are distributed geometrically spanning a range of $300-475 \mathrm{~K}$. The average replica exchange acceptance ratio is $42 \%$.

We simulate our systems using the GROMACS 2016.3 $3^{6,7}$ MD engine and PLUMED 2.3.1

for metadynamics calculations. ${ }^{8}$ Systems are propagated using the leap-frog algorithm with a 2 fs time step. The temperature of each replica is maintained using the Nosé-Hoover thermostat $^{9,10}$ with a 1 ps time constant. Electrostatic interactions are calculated using the 
particle-mesh Ewald method ${ }^{11}$ with a real space cutoff distance of $1 \mathrm{~nm}$. A $1 \mathrm{~nm}$ cutoff distance is used for the van der Waals interactions. 


\section{Supporting Figures}
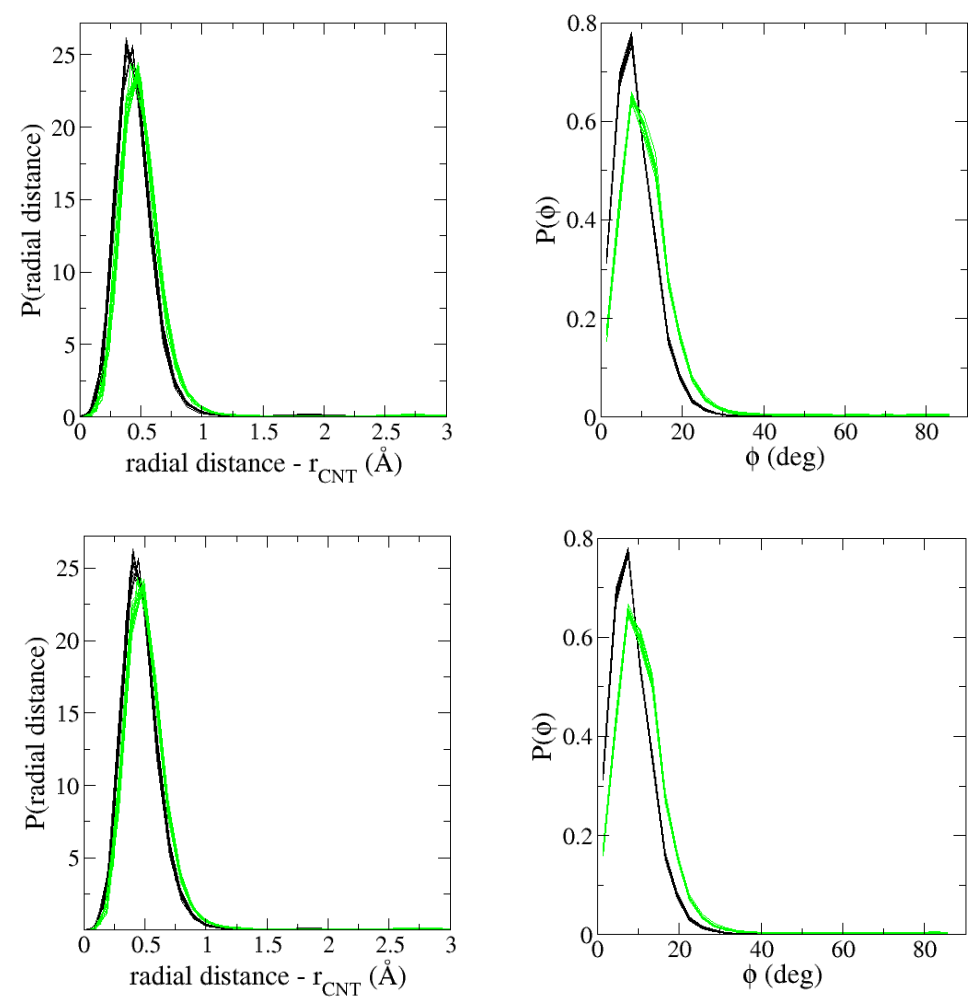

Figure S1: Normalized probability distribution of the distance between the geometric center of DNA bases and the CNT surface (left) and the stacking angle (right). The stacking angle $\phi$ is defined as $\phi=\arccos \left(\vec{n}_{\text {base }} \cdot \vec{n}_{C N T}\right)$, where $\vec{n}_{\text {base }}$ is a unit vector perpendicular to the plane of hexagon ring of aromatic DNA bases and $\vec{n}_{C N T}$ is a unit vector pointing radially outward from the center of the CNT toward the center of the hexagon ring. For a perfectly stacked base, $\phi$ is $0^{\circ}$. As we have the distance to CNT surface $<0.1 \mathrm{~nm}$ and stacking angle $<30^{\circ}$, we conclude that the bases are always adsorbed on CNT surface. Top row shows distance and angle distribution for D-DNA around each CNT and bottom row shows those for L-guanine (black) and L-thymine (green) around each CNTs. We note that in each of the panels, there are 17 green and 17 black curves representing separately each of the simulations. While the stacking angle and the distance is identical for D- and L-nucleotides, there are subtle differences between $\mathrm{G}$ (black) and $\mathrm{T}$ (green) residues. 

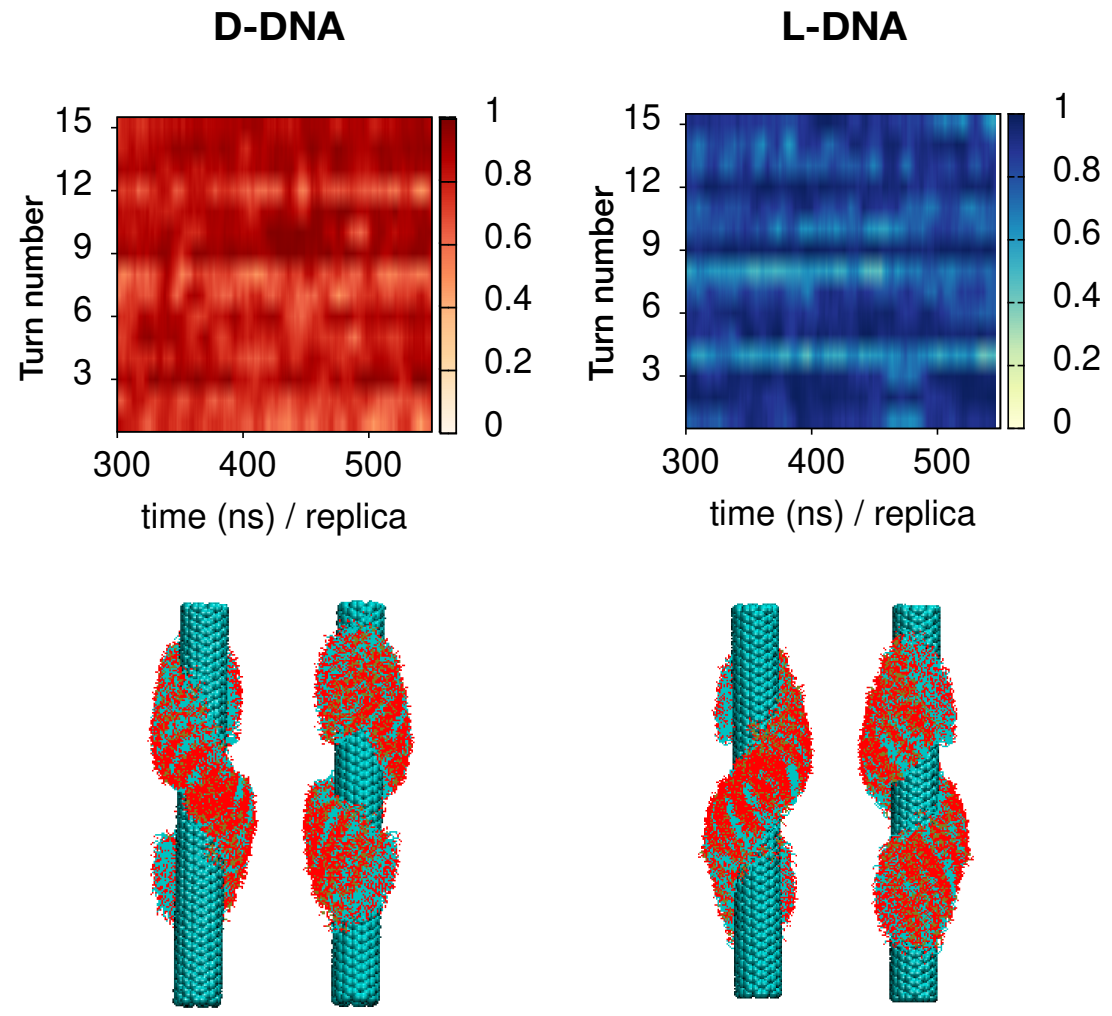

Figure S2: Average per-residue handedness of helical wraps of L- and D-forms of a random DNA sequence (TAGGCCAAGTGGATTGGA) around $(7,7) \mathrm{CNT}$ (top). Fraction of the time D-DNA residues wrap around CNTs in a left-handed fashion is shown in red hue. Fraction of the time L-DNA residues wrap around CNTs in a right-handed fashion is shown in blue hue. Clusters of contiguous helices of the DNA on the CNT for D-DNA and L-DNA (bottom). 

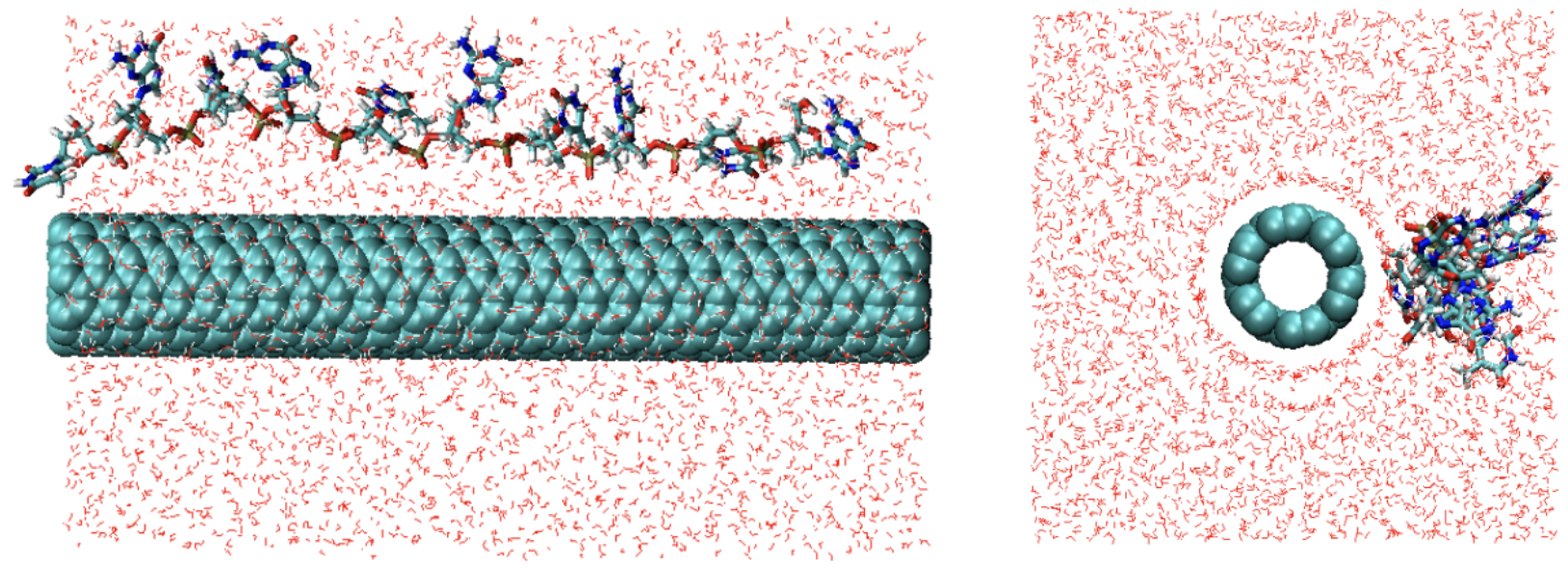

Figure S3: A representative example of starting configurations of the simulations performed in this work. 
D-DNA
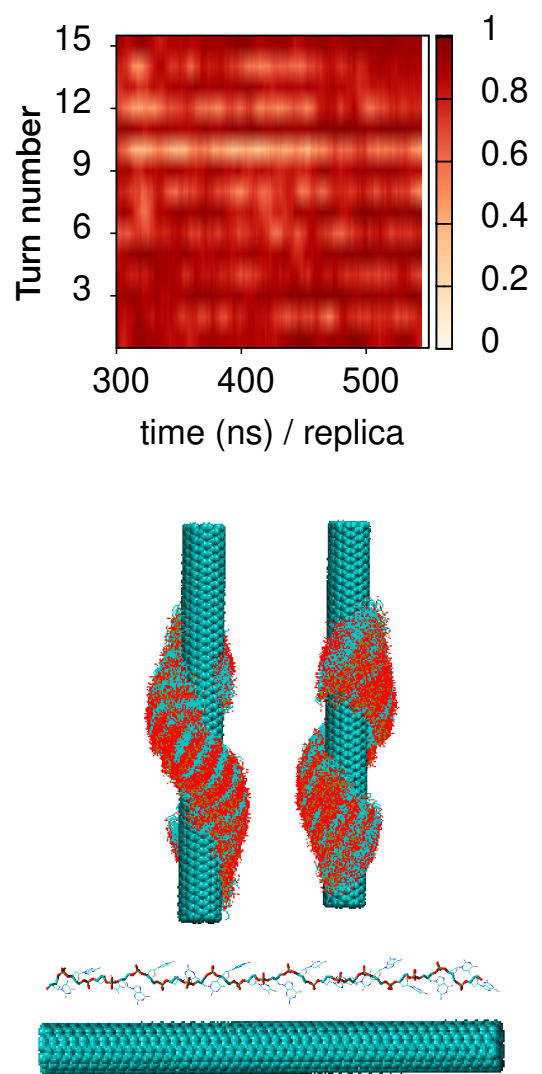

L-DNA
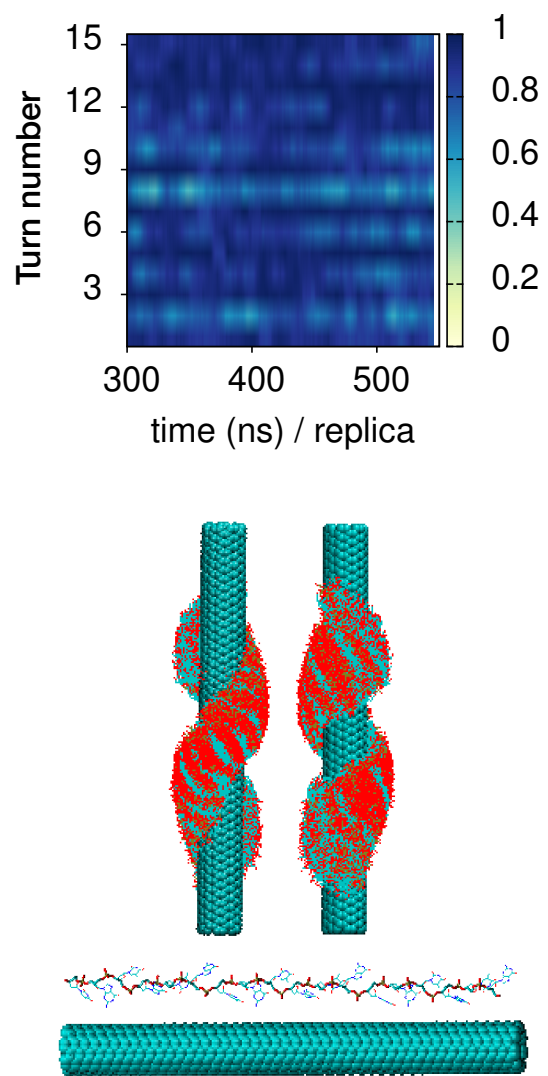

Figure S4: Average per-residue handedness of helical wraps of L- and D-forms of $(\mathrm{GT})_{9}$ around $(7,7)$ CNT (top). Fraction of the time D-DNA residues wrap around CNTs in a left-handed fashion is shown in red hue. Fraction of the time L-DNA residues wrap around CNTs in a right-handed fashion is shown in blue hue. Clusters of contiguous helices of the DNA on the CNT for D-DNA and L-DNA (middle). The initial configuration of (GT) $)_{9}$ DNA has the backbone dihedral angles of the " $\mathrm{S}_{1}$ " as specified by Klein and coworkers" (see the main text). 

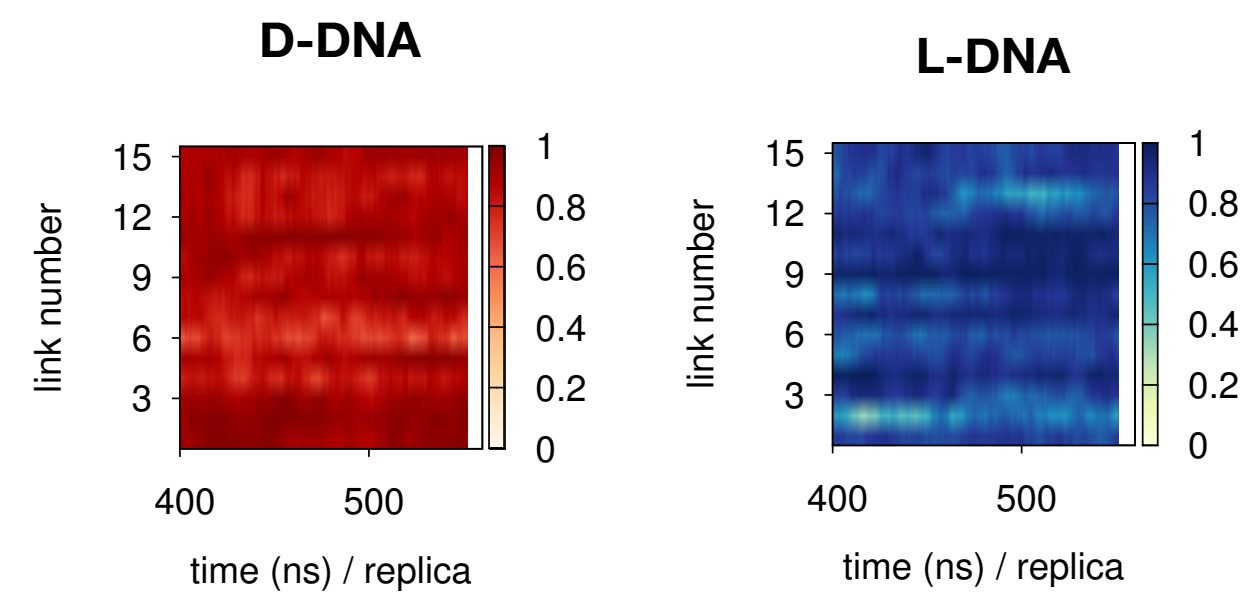

Figure S5: Average per-residue handedness of helical wraps of (L- and D-forms of) $\mathrm{GT}_{9}$ around a flexible $(7,7)$ CNT. Fraction of the time D-DNA residues wrap around CNTs in a left-handed fashion is shown in red hue. Fraction of the time L-DNA residues wrap around CNTs in a right-handed fashion is shown in blue hue. 
D-DNA

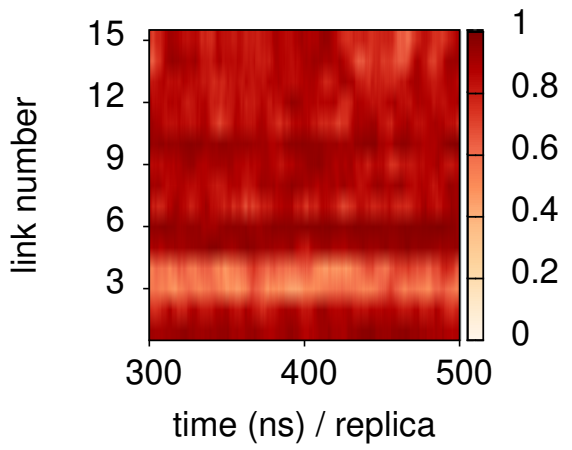

L-DNA

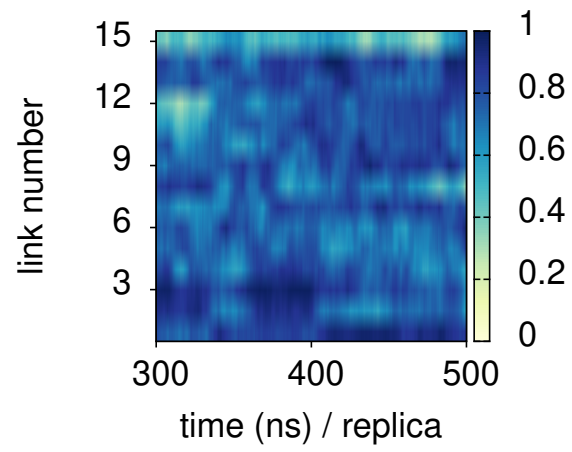

Figure S6: Average per-residue handedness of helical wraps of (L- and D-forms of) $\mathrm{GT}_{9}$ around for a larger diameter $(2 \mathrm{~nm}) \mathrm{CNT},(15,15) \mathrm{CNT}$. Fraction of the time D-DNA residues wrap around CNTs in a left-handed fashion is shown in red hue. Fraction of the time L-DNA residues wrap around CNTs in a right-handed fashion is shown in blue hue. 
(D-)DDD

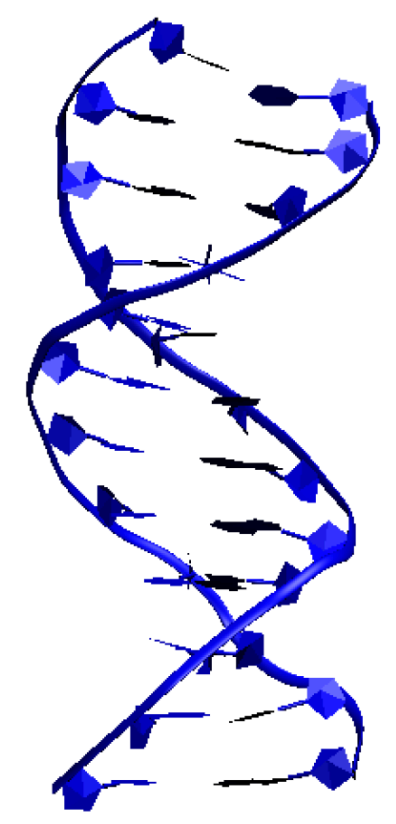

(L-)DDD

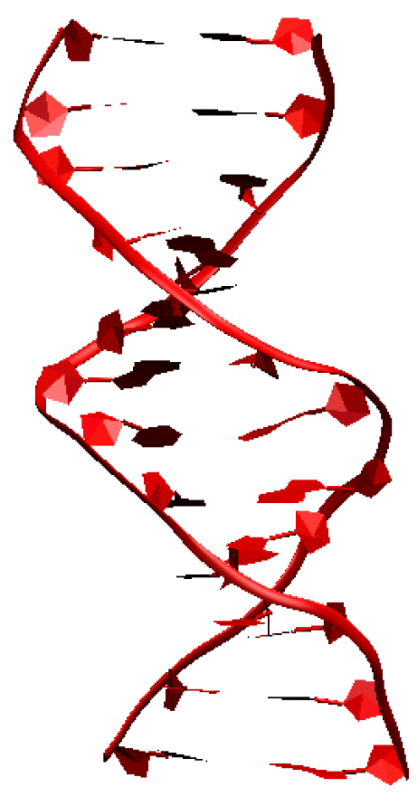

Figure S7: Comparison of the double-stranded helical structures of D-DNA and L-DNA. The DNA oligomer is Drew-Dickerson dodacemer (DDD). Melting curves are out of the scope of this work; however, in a work in progress, we show that the hybridization thermodynamics of DDD at its two opposite chiral realizations are identical. The only difference is the helical senses of their double-stranded helices, which are totally opposite of each other. Blue structure is a representative double stranded helical structure of the hybridized state of (D)DDD (right-handed helix) and the red structure is that of hybridized state of (L-)DDD, which is a left-handed helix. 


\section{References}

(1) Humphrey, W.; Dalke, A.; Schulten, K. J. Mol. Graphics 1996, 14, 33-38.

(2) Brooks, B. R.; Brooks, C. L.; MacKerell, A. D.; Nilsson, L.; Petrella, R. J.; Roux, B.; Won, Y.; Archontis, G.; Bartels, C.; Boresch, S.; Caflisch, A.; et al., J. Comput. Chem. 2009, 30, 1545-1614.

(3) Sugita, Y.; Okamoto, Y. Chem. Phys. Lett. 1999, 314, 141-151.

(4) Bonomi, M.; Parrinello, M. Phys. Rev. Lett. 2010, 104, 190601.

(5) Deighan, M.; Bonomi, M.; Pfaendtner, J. J. Chem. Theory Comput. 2012, 8, 21892192.

(6) Berendsen, H. J.; van der Spoel, D.; van Drunen, R. Comput. Phys. Commun. 1995, $91,43-56$.

(7) Hess, B.; Kutzner, C.; Van Der Spoel, D.; Lindahl, E. J. Chem. Theory Comput. 2008, $4,435-447$.

(8) Bonomi, M.; Branduardi, D.; Bussi, G.; Camilloni, C.; Provasi, D.; Raiteri, P.; Donadio, D.; Marinelli, F.; Pietrucci, F.; Broglia, R.; Parrinello, M. Comput. Phys. Commun. 2009, 180, 1961-1972.

(9) Nosé, S. Mol. Phys. 1984, 52, 255-268.

(10) Hoover, W. G. Phys. Rev. A 1985, 31, 1695.

(11) Essmann, U.; Perera, L.; Berkowitz, M. L.; Darden, T.; Lee, H.; Pedersen, L. G. J. Chem. Phys. 1995, 103, 8577-8593.

(12) Johnson, R. R.; Johnson, A. C.; Klein, M. L. Nano Lett. 2008, 8, 69-75. 